\title{
Anatomy of the hyper-runaway star LP 40-365 with Gaia
}

\author{
R. Raddi ${ }^{1 \star}$, M. A. Hollands ${ }^{2}$, B. T. Gänsicke 2 , D. M. Townsley ${ }^{3}$, J. J. Hermes ${ }^{4} \dagger$ \\ N. P Gentile Fusillo ${ }^{2}$, D. Koester ${ }^{5}$ \\ ${ }^{1}$ Dr. Remeis-Sternwarte, Friedrich Alexander Universität Erlangen-Nürnberg, Sternwartstr. 7, 96049 Bamberg, Germany \\ ${ }^{2}$ University of Warwick, Department of Physics, Gibbet Hill Road, Coventry, CV4 7AL, United Kingdom \\ ${ }^{3}$ University of Alabama, Department of Physics and Astronomy, Tuscaloosa, AL, USA \\ ${ }^{4}$ University of North Carolina, Department of Physics and Astronomy, Chapel Hill, NC - 27599-3255, US \\ ${ }^{5}$ Universität Kiel, Institut für Theoretische Physik und Astrophysik, 24098, Kiel, Germany
}

Accepted 2018 June 6. Received 2018 June 1; in original form 2018 April 25

\begin{abstract}
LP 40-365 (aka GD 492) is a nearby low-luminosity hyper-runaway star with an extremely unusual atmospheric composition, which has been proposed as the remnant of a white dwarf that survived a subluminous Type Ia supernova (SN Ia) in a single-degenerate scenario. Adopting the Gaia Data Release (DR2) parallax, $\varpi=1.58 \pm 0.03$ mas, we estimate a radius of $0.18 \pm 0.01 \mathrm{R}_{\odot}$, confirming LP 40-365 as a subluminous star that is $\simeq 15$ times larger than a typical white dwarf and is compatible with the SN Ia remnant scenario. We present an updated kinematic analysis, making use of the Gaia parallax and proper motion, and confirm that LP 40-365 is leaving the Milky Way at about 1.5 times the escape velocity of the Solar neighbourhood with a rest-frame velocity of $852 \pm 10 \mathrm{~km} \mathrm{~s}^{-1}$. Integrating the past trajectories of LP 40-365, we confirm it crossed the Galactic disc 5.0 $\pm 0.3 \mathrm{Myr}$ ago in the direction of Carina, likely coming from beneath the plane. Finally, we estimate that LP 40-365 was ejected from its progenitor binary with a velocity of at least $600 \mathrm{~km} \mathrm{~s}^{-1}$, which is compatible with theoretical predictions for close binaries containing a white dwarf and a helium-star donor.
\end{abstract}

Key words: stars: individual (GD 492) - supernova: general — white dwarfs subdwarfs - Galaxy: kinematics and dynamics

\section{INTRODUCTION}

There is general consensus that Type Ia supernovae (SN Ia) are the thermonuclear explosions of white dwarfs (Hillebrandt \& Niemeyer 2000). Although a common underlying mechanism makes $\mathrm{SNe}$ Ia standardisable candles for distances on cosmological scales (Riess et al. 1998; Perlmutter et al. 1999), their class is rich in peculiar objects, including the subluminous SNe Iax (Foley et al. 2013), the calcium-rich transients (Perets et al. 2011), and SNe .Ia (Bildsten et al. 2007).

Less settled is the discussion on the progenitors of SN Ia and their close relatives. Most scenarios assume that SN Ia originate from binary systems, with two fundamentally distinct channels: white dwarfs accreting from a non-degenerate companion (the single-degenerate channel) and mergers of white dwarfs pairs (the double-degenerate channel); for recent reviews, see Wang \& Han (2012); Maoz et al. (2014).

The chemically peculiar star, LP 40-365 (aka GD 492),

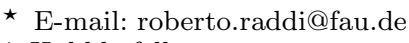

$\dagger$ Hubble fellow has been recently proposed by Vennes et al. (2017) as a partially burned white that survived a SNe Iax explosion (see Jordan et al. 2012; Kromer et al. 2013, 2015). The detection of a significantly super-Solar manganese-to-iron ratio (Raddi et al. 2018, hereafter Paper I) suggests that LP 40365 had a non-degenerate companion (Seitenzahl et al. 2013; Cescutti \& Kobayashi 2017). In the proposed scenario, LP 40-365 was unbound from the original binary and, due to its initially large orbital speed, it is now travelling at more than $500 \mathrm{~km} \mathrm{~s}^{-1}$ (corresponding to the measured radial velocity), becoming therefore a runaway $\operatorname{star}^{1}$.

In this Letter, we use the accurate parallax and proper motions available from the recent Gaia Data Release 2 (DR2; Gaia Collaboration et al. 2016, 2018) to carry out the first

\footnotetext{
1 Runaway stars are proposed to gain their momentum via ejection from binary SN explosions (Portegies Zwart 2000). Hyperrunaway stars are the fastest of this class, with velocities comparable to those of traditional hypervelocity stars, which are thought to form via multi-body interactions with super-massive black holes (Hills 1988; Brown 2015).
} 


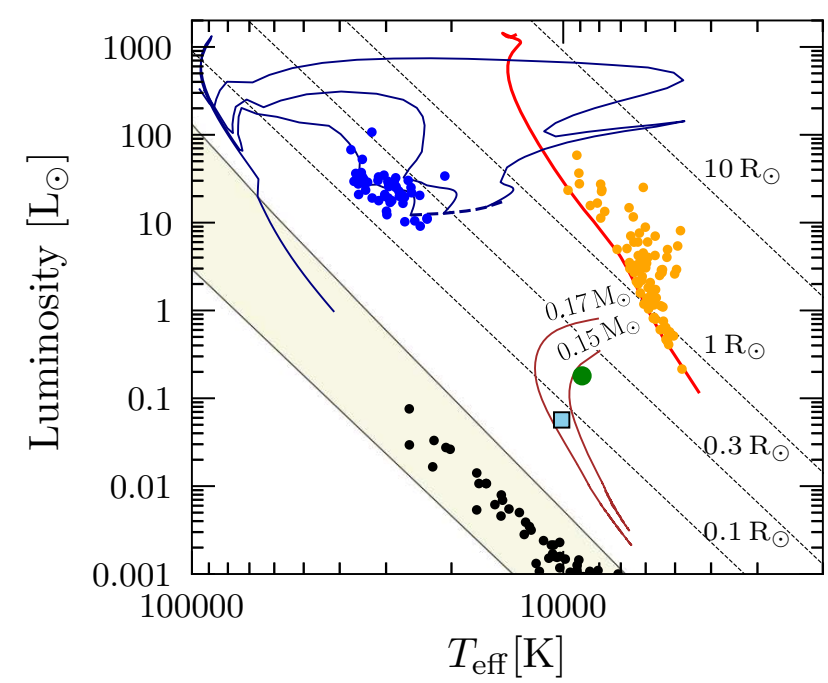

Figure 1. Hertzsprung-Russel diagram displaying LP 40-365, compared to various classes of stars. We plot the parameters based on our analysis including the Gaia data (green circle) and the Vennes et al. (2017) estimate (light-blue square), hot subdwarfs (blue dots; Lisker et al. 2005), main sequence stars (orange dots; Boyajian et al. 2013), and nearby white dwarfs (black dots; Giammichele et al. 2012). The proto- white dwarf sequence and the cooling tracks (Althaus et al. 2013) are shown for 0.15 and $0.17 \mathrm{M}_{\odot}$ white dwarfs (brown solid curves). For reference, we draw the canonical-mass white dwarf cooling sequence (beige-coloured strip; Fontaine et al. 2001), the $100 \mathrm{Myr}$ old main sequence (red curve; Choi et al. 2016), and the evolutionary tracks for $0.47,0.48$, and $0.50 \mathrm{M}_{\odot}$ hot subdwarfs (blue curves; Dorman et al. 1993). The luminosities for given stellar radii are shown as a function of $T_{\text {eff }}$ (dotted curves).

Table 1. Physical parameters of LP 40-365. The nominal values of $d, R$ and $M$ correspond to the median of the distributions, and the $1 \sigma$ uncertainty. The $5-95$ per cent range is also given below.

\begin{tabular}{cccccc}
\hline$T_{\text {eff }}^{1}$ & $\log g^{1}$ & $d$ & $R$ & $M$ & $L$ \\
\hline$(\mathrm{K})$ & $(\mathrm{cgs})$ & $(\mathrm{pc})$ & $\left(\mathrm{R}_{\odot}\right)$ & $\left(\mathrm{M}_{\odot}\right)$ & $(\mathrm{L} \odot)$ \\
\hline $8900 \pm 300$ & $5.50 \pm 0.25$ & $632 \pm 14$ & $0.18 \pm 0.01$ & $0.37_{-0.17}^{+0.29}$ & $0.18 \pm 0.01$ \\
& & $610-655$ & $0.16-0.20$ & $0.14-0.98$ & $0.17-0.19$ \\
\hline
\end{tabular}

${ }^{1}$ From Paper I.

detailed kinematic analysis of LP 40-365, which provides strong constraints on its physical parameters, its past trajectory, and the properties of the progenitor system.

\section{PHYSICAL PROPERTIES}

The precision of the Gaia parallax of LP40-365, $\varpi=$ $1.58 \pm 0.03$ mas, ensures a direct conversion between parallax and distance without significant loss of accuracy (Bailer-Jones et al. 2018, and references therein), placing LP $40-365$ at $632 \pm 14$ pc.

Scaling our best-fit model from Paper I to the Gaia magnitude, $G_{\mathrm{p}}=15.58 \mathrm{mag}$, which we corrected for the sightline interstellar extinction (0.02 mag; Green et al. 2018), we estimate the integrated flux density of LP 40-365 to be $f=1.45 \times 10^{-11} \mathrm{erg} / \mathrm{cm}^{2} / \mathrm{s}$. Thus, using the Gaia paral-

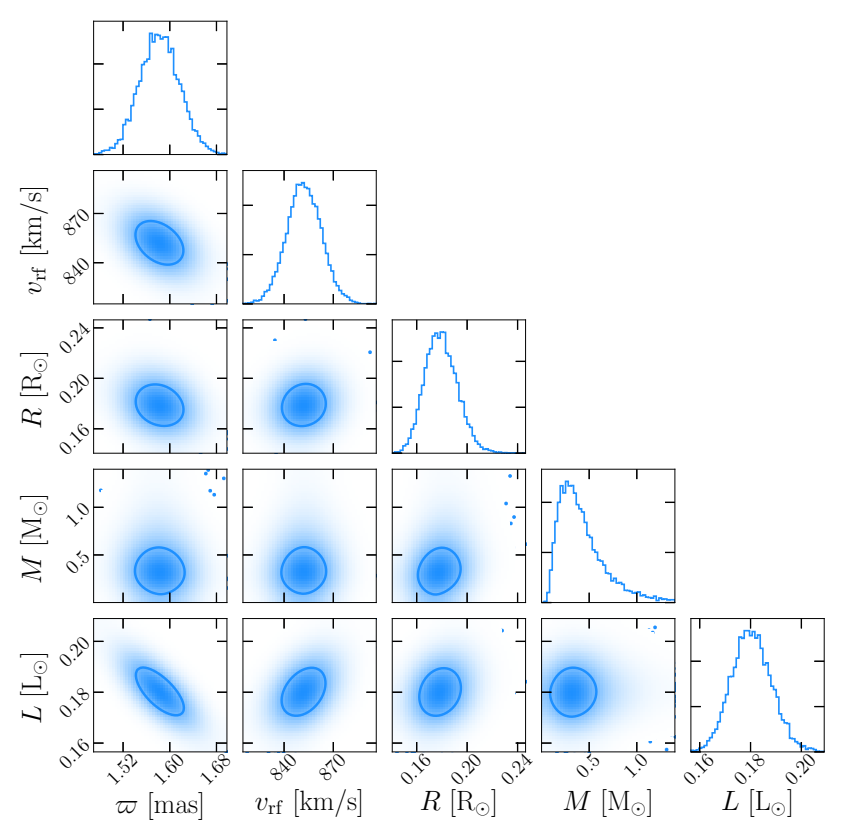

Figure 2. Corner plot displaying the correlation between the Gaia-DR2 parallax and the physical properties of LP 40-365. The $1 \sigma$ contours are shown on the correlation plots.

lax and the Stefan-Boltzmann law, the radius of LP 40-365 is constrained to $R=0.18 \pm 0.01 \mathrm{R} \odot$, accounting for the parallax and $T_{\text {eff }}$ uncertainties. This result contrasts with the estimate of $0.07 \mathrm{R}_{\odot}$, obtained by Vennes et al. (2017) via interpolation of their $T_{\text {eff }}$ and $\log g$ with cooling models for lowmass helium-core white dwarfs (Althaus et al. 2013). On the Hertzsprung-Russel diagram (Fig. 1), our new results place LP 40-365 at a cooler and brighter location with respect to the parameters of Vennes et al. (2017).

Combining our radius estimate with the surface gravity derived from the spectral fit in Paper I implies the mass of LP 40-365 is constrained as $M \propto g R^{2}=0.37_{-0.17}^{+0.29} \mathrm{M}_{\odot}$, with the 5-95 per cent confidence range between $M=$ $0.14-0.98 \mathrm{M}_{\odot}$. We note that LP 40-365 does not match the radius-luminosity relation of main sequence stars, as it is two orders of magnitude less luminous than stars of similar $T_{\text {eff }}$ (A-type stars), while it is hotter than mainsequence stars of similar radii (M-type dwarfs). LP 40-365 also diverges from the mass-radius relation of both canonical white dwarfs (Tremblay et al. 2017) and low-mass heliumcore white dwarfs (Althaus et al. 2013), with a composition clearly excluding a membership to the latter class of stars.

We will discuss the present appearance of LP 40-365 with reference to its evolutionary status in Section 4. The physical parameters are summarised in Table 1, while their correlation with $\varpi$ is shown in Fig. 2.

\section{KINEMATIC ANALYSIS}

The high precision of Gaia parallaxes enables measuring the total rest-frame velocity of LP 40-365 with no a priori assumption. Taking into account the correlation between the astrometric quantities, and using the radial velocity from Paper I, $v_{\text {rad }}=499 \pm 6 \mathrm{kms}^{-1}$, with the Galactic parame- 

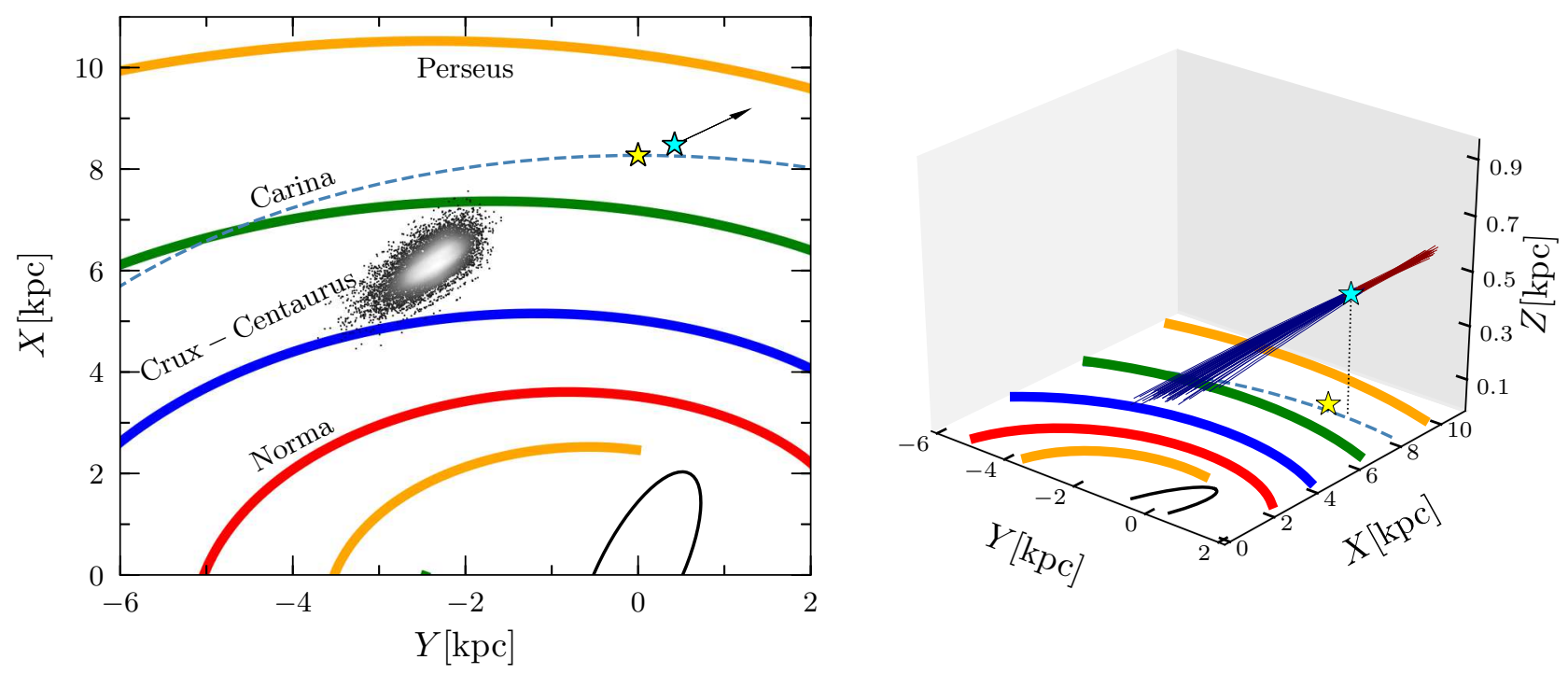

Figure 3. Simulated trajectories of LP 40-365 in the Milky Way potential. In both panels, the Sun and LP 40-365 are represented as a yellow and a cyan star, respectively, while the spiral arms and the Galactic bar are shown as colored and black curves (Vallée 2008), respectively. The Solar circle is traced by a dashed curve. Left panel: The reference frame follows the standard left-handed Galactocentric convention, with the Galactic centre at $X, Y=(0,0)$ and the $Y$-axis oriented in the direction of the Galactic rotation. The arrow shows the mean escape direction of LP 40-365. The cloud of dots represents the $X-Y$ coordinates of the trajectories crossing the Galactic plane $(Z=0)$. Right panel: Three-dimensional representation of a random sample of simulated trajectories. Past and future trajectories are plotted as dark-blue and red curves, respectively. The vertical dotted line shows the distance $Z$ of LP 40-365 from the plane. Note the scale on the $Z$ axis is expanded with respect to the $X-Y$ plane to enhance the visualization.

ters described below, we estimate the rest-frame velocity as $v_{\mathrm{rf}}=852 \pm 10 \mathrm{~km} \mathrm{~s}^{-1}$, making it the fastest known hyperrunaway star that is the nearest to the Sun. At this remarkably large speed, LP 40-365 exceeds the Galactic escape velocity $\left(520-533 \mathrm{~km} \mathrm{~s}^{-1}\right.$ in the Solar neighbourhood; Piffl et al. 2014; Williams et al. 2017, or $\approx 550 \mathrm{~km} \mathrm{~s}^{-1}$ as for the adopted Galactic model), thus it is confirmed as gravitationally unbound from the Milky Way.

To investigate the space motion of LP 40-365, we used the Galactic orbit integrator implemented in the PYTHON package GALPY ${ }^{2}$ (Bovy 2015). We set up the Galactic potential described in Bovy \& Rix (2013), which consists of three components (bulge, disk, and halo), to which we added a central black hole of $4 \times 10^{6} \mathrm{M}_{\odot}$ (Bovy 2015). In this model, following Schönrich (2012), the Sun is placed at $R_{0}=8.27 \pm 0.29 \mathrm{kpc}$ from the Galactic centre and the Milky Way rotation speed at the Solar circle is $V_{\mathrm{c}}=238 \pm 9 \mathrm{~km} \mathrm{~s}^{-1}$, while the peculiar motion of the Sun in the Local Standard of Rest is $\left(U_{\odot}, V_{\odot}, W_{\odot}\right)=(11.1,12.24,7.25) \mathrm{km} \mathrm{s}^{-1}$, from Schönrich et al. (2010). Our simulations take into account the statistical and systematic uncertainties quoted in the original works. The boundary conditions correspond to the Gaia DR2 observables, $\alpha, \delta, \mu_{\alpha}, \mu_{\delta}$, and $\varpi$, along with $v_{\text {rad }}$ from Paper I. We sampled the boundary conditions via a Monte Carlo method, taking into account the Gaia covariance matrix and assuming Gaussian distributions for the Galactic model parameters. We back-traced the trajectories for $250 \mathrm{Myr}$ in the past, i.e. the timescale of one Solar orbit around the Milky Way at the Galatocentric distance of the Sun. We display a representative set of trajectories in Fig. 3.
From this simulation, we find that LP 40-365 crossed the Galactic plane in the inter-arm region between the CruxCentaurus and the Carina spiral arms, at $4.2 \pm 0.5 \mathrm{kpc}$ from the present position of Sun, corresponding to a Galactocentric radius $R_{\mathrm{G}}=6.0 \pm 0.3 \mathrm{kpc}$. At its speed, LP 40-365 has travelled for $5.0 \pm 0.3 \mathrm{Myr}$ to reach its current location after it crossed the Galactic disc. We do not attempt to associate LP 40-365 to any known Galactic structure along its trajectory (e.g. clusters, spiral arms, or stellar streams), given that its age is not sufficiently well constrained. However, we note that the flight time from a Galactocentric distance of $100 \mathrm{kpc}(Z=-7.3 \pm 0.5 \mathrm{kpc})$ is $\approx 140 \mathrm{Myr}$, setting an upper limit on the cooling age, if LP 40-365 was ejected from the Galactic halo. We speculate that its membership to known structures could be investigated in future, when evolutionary models will become available. An origin in Galactic centre or in the Magellanic Clouds can definitely be excluded.

In Fig. 4, we show the evolution of positional and kinematic parameters of LP 40-365. We note the asymptotic behaviour of $Z, R_{\mathrm{G}}$, and $d$, which is typical of open trajectories. We also note that LP 40-365 was accelerated while crossing the Galactic disc $\approx 5 \mathrm{Myr}$ ago and it is slowing down while leaving the Milky Way. All the future trajectories reach $R_{\mathrm{G}}=100 \mathrm{kpc}$ (not plotted in Fig. 4 ) in $\approx 130 \mathrm{Myr}$.

The simulated trajectories have small pitch angles with respect to the plane, $\gamma \approx 5.6 \mathrm{deg}$, potentially implying a boost from the Galactic rotation (see Kenyon et al. 2014) if the ejection occurred in the Galactic disc. The ejection velocity from the putative progenitor system, $v_{\mathrm{ej}}$, could be estimated from $v_{\mathrm{rf}}$ via:

$v_{\mathrm{rf}}^{2}=v_{\mathrm{ej}}^{2}+V_{\mathrm{c}}^{2}+2 v_{\mathrm{ej}} V_{\mathrm{c}} \cos \beta \cos \gamma$,

2 https://github.com/jobovy/galpy ) 

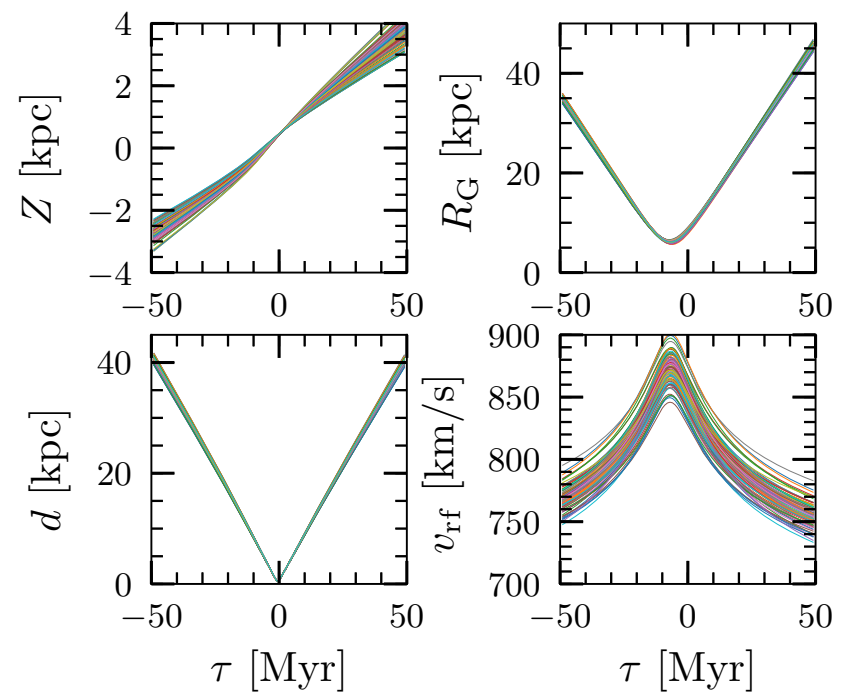

Figure 4. Evolution of positional and kinematic parameters for a representative sample of LP 40-365 trajectories, plotted as function of the flight time, $\tau$. Clockwise from top left: Galactic $Z$ coordinate; Galactocentric radius $R_{\mathrm{G}}$; rest frame velocity $v_{\mathrm{rf}}$; Heliocentric distance $d . \tau=0$ corresponds to the Gaia DR2 epoch.

where $v_{\mathrm{c}}$ is the rotational velocity of the progenitor in the Milky Way at the moment of the explosion, and $\gamma$ is the angle between $v_{\mathrm{c}}$ and $v_{\mathrm{ej}}$ in the Galactic plane. However, we stress that Eq. 1 only holds if LP 40-365's progenitor exploded close to the Galactic plane. In this case, we would have that $v_{\mathrm{ej}} \sim 600 \mathrm{~km} \mathrm{~s}^{-1}$. Given that evolutionary timescales of a peculiar white dwarfs such as LP 40-365 are yet unconstrained, it cannot be excluded with certainty that the progenitor exploded at several kiloparsecs from the Galactic centre. Hence, the contribution to $v_{\mathrm{rf}}$ due to the Galactic rotation would be negligible for a halo progenitor, so that $v_{\mathrm{ej}} \approx v_{\mathrm{rf}}$.

\section{PROPERTIES OF THE BINARY PROGENITOR}

Given that LP 40-365 is a single star (Vennes et al. 2017; Raddi et al. 2018), if it is the partly-burnt remnant of a SN Iax, the progenitor system must have been separated as consequence of mass loss caused by the SN explosion.

Following Hills (1983), under the assumption of instantaneous mass loss, a binary system breaks apart if it experiences a minimum mass loss, $\delta M$, defined as:

$\left\langle\left(\frac{\delta M}{M_{\text {prog }}+M_{\text {donor }}}\right)_{\min }\right\rangle=0.5 \times\left[1-\left(\frac{v_{\text {kick }}}{v_{\text {orbit }}}\right)^{2}\right]$,

where $M_{\text {prog }}$ and $M_{\text {donor }}$ are the mass of progenitor and donor stars, respectively, and $v_{\text {kick }}$ is a velocity kick that could arise from e.g. asymmetric explosions (Jordan et al. 2012).

In Paper I, we brought evidence in favour of a singledegenerate scenario via the detection of a super-Solar manganese-to-iron ratio, which requires a $M_{\text {prog }}>1.2 \mathrm{M}_{\odot}$ to synthesise manganese (Seitenzahl et al. 2013). Thus, considering the simplest case, with negligible mass-loss from the donor star (as shown by Marietta et al. 2000; Pan et al.
2012; Liu et al. 2012, 2013, for non-giant companions) and no birth kick $\left(v_{\text {kick }}=0\right)$, we have that the minimum possible mass of the donor star is $M_{\text {donor }}=0.86_{-0.58}^{+0.34} \mathrm{M}_{\odot}$. Hence, the donor star can be constrained to $\leq 1.32 \mathrm{M}_{\odot}$ at the 95 per cent confidence limit. Numerical simulations of subluminous SN explosions that form partly-burnt remnants also produce $v_{\text {kick }}$ of up to a few hundred $\mathrm{km} \mathrm{s}^{-1}$, although debated (Jordan et al. 2012; Kromer et al. 2013), that would enable to unbind binaries with even more massive donors.

Considering possible ejection velocities at the moment of explosion, $v_{\mathrm{ej}} \lesssim v_{\mathrm{rf}}=600-800 \mathrm{~km} \mathrm{~s}^{-1}$, the orbital period of a near-Chandrasekhar mass white dwarf with a donor star between $0.3-1.2 \mathrm{M}_{\odot}$ is in the range of $30 \mathrm{~min}$ to $1 \mathrm{hr}$. Binaries leading to such tight configurations have been theoretically identified in the helium star donor channel of the singledegenerate scenario (Wang et al. 2009, 2014). These systems are proposed as a leading mechanism behind subluminous SNe Iax (Jha 2017; McCully et al. 2014), which could also have an important contribution to the population of Galactic hyper-runaway stars (Justham et al. 2009; Wang \& Han 2009). They include either massive helium core- or shellburning donor stars (up to $2.5 \mathrm{M}_{\odot}$ ), down to canonical mass hot subdwarfs $\left(\approx 0.47 \mathrm{M}_{\odot} ;\right.$ Heber 2016$)$. As already noted by Vennes et al. (2017), the hypervelocity hot subdwarf US 708 (Geier et al. 2015) and LP 40-365 could represent two sides of the same coin: a donor star and an unburnt remnant surviving a SN Ia in a close binary. US 708 is suggested to have a mass of $0.3 \mathrm{M}_{\odot}$, and it is proposed to have originated as the donor in an ultra compact 10-min binary exploded as sub-Chandrasekhar mass SN. LP 40-365, instead, is unique in its kind, as it is the first surviving white dwarf fitting with this scenario. Gaining a speed as large as that of of US 708 $\left(\sim 1200 \mathrm{~km} \mathrm{~s}^{-1}\right.$; Geier et al. 2015), the subdwarf donor of LP 40-365 could have escaped the Milky Way long time ago.

However, we note that the hot subdwarf donor scenario may not entirely fit with LP 40-365, because lowmass donors $\left(\lesssim 0.8 \mathrm{M}_{\odot}\right)$ are not expected to lead to near-Chandrasekhar mass SN Ie, due to unstable helium-shell burning (Piersanti et al. 2014; Brooks et al. 2015). More massive donors $\left(0.8-1.7 \mathrm{M}_{\odot}\right.$; Piersanti et al. 2014; Brooks et al. 2017), instead, could lead to nearChandrasekhar mass explosions, after accreting sufficient mass on to the white dwarf. While such progenitors could be identifiable as helium-novae, or super soft X-ray sources, some detached candidates of this SN channel have been proposed, e.g. KPD 1930+2752 (Maxted et al. 2000; Geier et al. 2007). Although this scenario is theoretically appealing, very compact systems like these remain, so far, rare (just three are known with $P_{\text {orb }}<90$ min; Vennes et al. 2012; Kupfer et al. 2017a,b) and their fate is still debated.

In this context, Shen et al. (2018) have recently identified three hyper-runaway stars classified as "expanded" white dwarfs, which are proposed as former donor stars in doubledegenerate systems. These stars would now have larger luminosities than normal white dwarfs due to several effects, such as dynamical interactions within the progenitor binary, impact of the SN ejecta, and accretion of radioactive ${ }^{56} \mathrm{Ni}-$ rich material, all of which contributed to (partially) lift the core degeneracy and expand the envelope. Although having larger $v_{\mathrm{rf}}$, redder colours, and a likely carbon-oxygen atmosphere, Shen et al. (2018) proposed these new stars and LP 40-365 to form a short-lived heterogeneous class of 
stars that would eventually re-join the white dwarf cooling sequence. As the peculiar atmospheric composition of LP 40-365 remains the strongest connection to the partlyburned remnants of SN Iax, we note that such objects would also expand due to internal adjustments of the stellar structure after the explosion (Kromer et al. 2013; Shen \& Schwab 2017). Further theoretical investigations concerning the evolutionary timescales are crucial, given the small parameter space tested in the literature.

Finally, another physical constraint worth comparing is the rotational velocity of LP 40-365 to that of US 708. The hot subdwarf shows a high rotational velocity $\left(115 \mathrm{~km} \mathrm{~s}^{-1}\right.$; Geier et al. 2015), consistent with it being tidally locked at an orbital period before detonation of roughly $10 \mathrm{~min}$. US 708 is best explained as the donor to an exploded system that has not significantly evolved in size since the event.

On the other hand, we do not measure any rotational broadening for LP 40-365, and can only establish an upper limit on the rotational velocity of $50 \mathrm{~km} \mathrm{~s}^{-1}$ (Paper I). To have been ejected from the binary with $v_{\mathrm{ej}}>600 \mathrm{~km} \mathrm{~s}^{-1}$ it is likely that LP 40-365 became unbound from a tight system with an orbital period shorter than $1 \mathrm{hr}$. Tides are likely to synchronize the rotation of both components of such short orbital periods (Fuller \& Lai 2012).

The rotation velocity of a $0.18 \mathrm{R}_{\odot}$ star rotating faster than $1 \mathrm{hr}$ would exceed $200 \mathrm{~km} \mathrm{~s}^{-1}$, which we would be able to detect in LP 40-365 for all inclinations higher than roughly 20 deg. However, if the progenitor of LP 40-365 were a massive white dwarf rotating at the previous orbital period, its radius will have increased more than a factor of 20 to match the size we observe today; its rotation would have likely slowed considerably to conserve angular momentum. Therefore the relatively slow rotation velocity adds another line of evidence to LP 40-365 being the partly burnt remnant of a sub-luminous supernova and not the donor.

\section{SUMMARY AND CONCLUSIONS}

LP 40-365 is a hyper-runaway star, which is the first example of a possible SN Iax survivor, i.e. a partly burnt white dwarf that is enriched with nuclear ashes (Vennes et al. 2017; Raddi et al. 2018). Here, we have presented a detailed analysis of its physical and kinematic properties, making use of the recent Gaia-DR2 astrometry. At a distance of $632 \pm 14$ pc, LP 40-365 is the nearest hyper-runaway star to the Sun that is unbound from the Galaxy, having a restframe velocity of $852 \pm 10 \mathrm{~km} \mathrm{~s}^{-1}$. We confirm it as a subluminous star, with a radius of $0.18 \pm 0.01 \mathrm{R}_{\odot}$ and a mass of $0.37_{-0.17}^{+0.29} \mathrm{M}_{\odot}$, matching the partly-burnt white dwarf hypothesis.

We simulated the past trajectory of LP 40-365 in the Milky Way potential, finding it crossed the Galactic disc $\approx 5 \mathrm{Myr}$ ago in the direction of Carina at a Galactocentric radius of $\approx 6.5 \mathrm{kpc}$, i.e. $\approx 4.2 \mathrm{kpc}$ from the Sun's current position. With a cooling age possibly as large as $\sim 100 \mathrm{Myr}$, LP 40-365 could have been ejected from the Galactic halo at a few kpc below the plane. From the constraint we have on LP 40-365's mass, we suggest its progenitor may have been a short-period (30-60 min) binary with a donor star of $0.8-$ $1.32 \mathrm{M}_{\odot}$. The ejection velocities from such tight binaries are sufficient to accelerate LP 40-365 and similar objects to and beyond the Galactic escape velocity.

With the high quality data of Gaia DR2, we expect that more candidates of partially burned remnants and/or donor stars ejected from SN Ia will be identified, helping to fill in the gaps between theoretical predictions and unusual stars such as LP 40-365 and those found by Shen et al. (2018).

\section{ACKNOWLEDGEMENTS}

We thank U. Heber for useful discussions. R.R. acknowledges funding by the German Science foundation (DFG) through grants HE1356/71-1 and IR190/1-1. The research leading to these results has received funding from the European Research Council under the European Union's Seventh Framework Programme (FP/2007-2013) / ERC Grant Agreement n. 320964 (WDTracer). Support for this work was provided by NASA through Hubble Fellowship grant \#HST-HF2-51357.001-A, awarded by the Space Telescope Science Institute, which is operated by the Association of Universities for Research in Astronomy, Incorporated, under NASA contract NAS5-26555.

This work has made use of data from the European Space Agency (ESA) mission Gaia (https://www.cosmos.esa.int/gaia), processed by the Gaia Data Processing and Analysis Consortium (DPAC, https://www . cosmos.esa.int/web/gaia/dpac/consortium). Funding for the DPAC has been provided by national institutions, in particular the institutions participating in the Gaia Multilateral Agreement.

\section{REFERENCES}

Althaus L. G., Miller Bertolami M. M., Córsico A. H., 2013, A\&A, 557, A19

Bailer-Jones C. A. L., Rybizki J., Fouesneau M., Mantelet G., Andrae R., 2018, preprint, (arXiv:1804.10121)

Bildsten L., Shen K. J., Weinberg N. N., Nelemans G., 2007, ApJ, 662, L95

Bovy J., 2015, ApJS, 216, 29

Bovy J., Rix H.-W., 2013, ApJ, 779, 115

Boyajian T. S., et al., 2013, ApJ, 771, 40

Brooks J., Bildsten L., Marchant P., Paxton B., 2015, ApJ, 807,74

Brooks J., Schwab J., Bildsten L., Quataert E., Paxton B., 2017, ApJ, 834, L9

Brown W. R., 2015, ARA\&A, 53, 15

Cescutti G., Kobayashi C., 2017, A\&A, 607, A23

Choi J., Dotter A., Conroy C., Cantiello M., Paxton B., Johnson B. D., 2016, ApJ, 823, 102

Dorman B., Rood R. T., O'Connell R. W., 1993, ApJ, 419, 596

Foley R. J., et al., 2013, ApJ, 767, 57

Fontaine G., Brassard P., Bergeron P., 2001, PASP, 113, 409

Fuller J., Lai D., 2012, MNRAS, 421, 426

Gaia Collaboration et al., 2016, A\&A, 595, A1

Gaia Collaboration Brown A. G. A., Vallenari A., Prusti T., de Bruijne J. H. J., Babusiaux C., Bailer-Jones C. A. L., 2018, preprint, (arXiv:1804.09365)

Geier S., Nesslinger S., Heber U., Przybilla N., Napiwotzki R., Kudritzki R.-P., 2007, A\&A, 464, 299

Geier S., et al., 2015, Science, 347, 1126

Giammichele N., Bergeron P., Dufour P., 2012, ApJS, 199, 29

Green G. M., et al., 2018, preprint, (arXiv:1801.03555) 
Heber U., 2016, PASP, 128, 082001

Hillebrandt W., Niemeyer J. C., 2000, ARA\&A, 38, 191

Hills J. G., 1983, ApJ, 267, 322

Hills J. G., 1988, Nature, 331, 687

Jha S. W., 2017, Type Iax Supernovae. p. 375, doi:10.1007/978-3-319-21846-5_42

Jordan IV G. C., Perets H. B., Fisher R. T., van Rossum D. R., 2012, ApJ, 761, L23

Justham S., Wolf C., Podsiadlowski P., Han Z., 2009, A\&A, 493, 1081

Kenyon S. J., Bromley B. C., Brown W. R., Geller M. J., 2014, ApJ, 793, 122

Kromer M., et al., 2013, MNRAS, 429, 2287

Kromer M., et al., 2015, MNRAS, 450, 3045

Kupfer T., et al., 2017a, preprint, (arXiv:1710.07287)

Kupfer T., et al., 2017b, ApJ, 835, 131

Lisker T., Heber U., Napiwotzki R., Christlieb N., Han Z., Homeier D., Reimers D., 2005, A\&A, 430, 223

Liu Z. W., Pakmor R., Röpke F. K., Edelmann P., Wang B., Kromer M., Hillebrandt W., Han Z. W., 2012, A\&A, 548, A2

Liu Z.-W., et al., 2013, ApJ, 774, 37

Maoz D., Mannucci F., Nelemans G., 2014, A\&ARv, 52, 107

Marietta E., Burrows A., Fryxell B., 2000, ApJS, 128, 615

Maxted P. F. L., Marsh T. R., North R. C., 2000, MNRAS, 317, L41

McCully C., et al., 2014, Nature, 512, 54

Pan K.-C., Ricker P. M., Taam R. E., 2012, ApJ, 750, 151

Perets H. B., Gal-yam A., Crockett R. M., Anderson J. P., James P. A., Sullivan M., Neill J. D., Leonard D. C., 2011, ApJ, 728, L36

Perlmutter S., et al., 1999, ApJ, 517, 565

Piersanti L., Tornambé A., Yungelson L. R., 2014, MNRAS, 445,3239

Piffl T., et al., 2014, A\&A, 562, A91

Portegies Zwart S. F., 2000, ApJ, 544, 437

Raddi R., Hollands M. A., Koester D., Gänsicke B. T., Gentile Fusillo N. P., Hermes J. J., Townsley D. M., 2018, ApJ, 858, 3

Riess A. G., et al., 1998, AJ, 116, 1009

Schönrich R., 2012, MNRAS, 427, 274

Schönrich R., Binney J., Dehnen W., 2010, MNRAS, 403, 1829

Seitenzahl I. R., Cescutti G., Röpke F. K., Ruiter A. J., Pakmor R., 2013, A\&A, 559, L5

Shen K. J., Schwab J., 2017, ApJ, 834, 180

Shen K. J., et al., 2018, preprint, (arXiv:1804.11163)

Tremblay P.-E., et al., 2017, MNRAS, 465, 2849

Vallée J. P., 2008, AJ, 135, 1301

Vennes S., Kawka A., O’Toole S. J., Németh P., Burton D., 2012, ApJ, 759, L25

Vennes S., Nemeth P., Kawka J. R., Thorstensen J. R., Khalack V., Ferrario L., Alper E. H., 2017, Science, 357, 680

Wang B., Han Z., 2009, A\&A, 508, L27

Wang B., Han Z., 2012, New Astron. Rev., 56, 122

Wang B., Meng X., Chen X., Han Z., 2009, MNRAS, 395, 847

Wang B., Meng X., Liu D.-D., Liu Z.-W., Han Z., 2014, ApJ, 794, L28

Williams A. A., Belokurov V., Casey A. R., Evans N. W., 2017, MNRAS, 468, 2359

This paper has been typeset from a $\mathrm{T}_{\mathrm{E}} \mathrm{X} / \mathrm{LAT}_{\mathrm{E}} \mathrm{X}$ file prepared by the author. 